\title{
A dual interpolation boundary face method for 3D elasticity
}

\section{Jianming Zhang*, Chuanming Ju, Pihua Wen, Xiaomin Shu, WeiCheng Lin, Baotao Chi}

State Key Laboratory of Advanced Design and Manufacturing for Vehicle Body, College of Mechanical and Vehicle Engineering, Hunan University, Changsha 410082, China

Correspondence to: Jianming Zhang

College of Mechanical and Vehicle Engineering, Hunan University, Changsha 410082, China

Telephone: +86-731-88823061

E-mail: zhangjm@hnu.edu.cn

\begin{abstract}
The dual interpolation boundary face method (DiBFM) proposed recently has been successfully applied to solve various problems in two dimensions in this paper. Compared with the conventional boundary element method (BEM), it has been proved that the DiBFM has the advantages of higher accuracy, convergence rate and computational efficiency. In addition, the DiBFM is able to unify the conforming and nonconforming elements in the BEM implementation, as well as to approximate both continuous and discontinuous stress fields. Moreover, there are no geometric errors by the DiBFM in the computational process. In this paper, the DiBFM is extended successfully to solve the elasticity problems in three-dimensions (3D) with formulations of DiBFM derived in details. A number of numerical examples are presented in order to validate the accuracy and convergence rate of the proposed method.
\end{abstract}

Keywords: Dual interpolation boundary face method; 3D elasticity problems; boundary element method; moving least-squares approximation.

\section{Introduction}

Recently, the dual interpolation boundary face method has been successfully applied to solve various 2D problems, such as potential [1], elasticity [2], thin-walled structures [3], contact [4], and V-shaped notch problems [5]. This is due to the fact that the DiBFM can achieve higher accuracy and convergence rates than the conventional BEM for most cases [1]. These 
advantages stem from the fact that the DiBFM is coupled between the dual interpolation method and the boundary face method.

The dual interpolation method specifically refers to the first-layer interpolation and the second-layer interpolation, while the element in the method is called dual interpolation element. The dual interpolation element consists of source and virtual nodes, as shown in Fig.1. When ignoring virtual nodes, it becomes a conventional discontinuous element. If both the virtual nodes and the source nodes are considered, then it amounts to a standard continuous element. In this manner, the continuous and discontinuous elements schemes can be unified by the dual interpolation element [1, $6]$. Thus, the geometric corner problems $[2,7,8]$ and the mesh generation can be treated easily $[9$, 10].

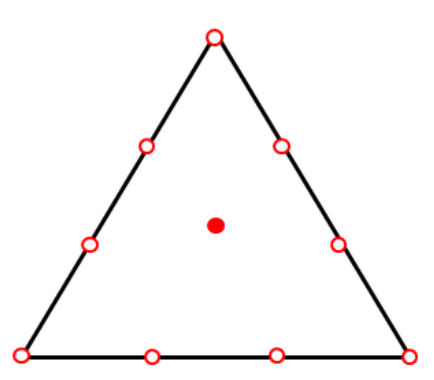

o virtual node

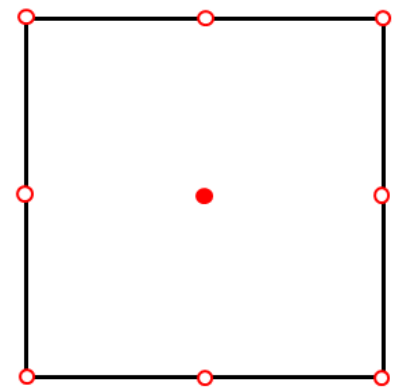

- source node

Fig.1. Dual interpolation elements in 3D problems.

The first-layer interpolation is similar to the interpolation in a conventional continuous boundary element. Namely, both source nodes and virtual nodes in the dual interpolation elements are used for interpolation. By adding the virtual nodes in dual interpolation elements, the interpolation order of the dual interpolation elements is increased obviousely, comparing to the interpolation function order of the conventional discontinuous elements which only use the source nodes $[1,4]$. This results in a significant improvement for interpolation accuracy.

The second-layer interpolation is constructed by the moving least square (MLS) approximation and this approximation is employed to condense the degrees of freedom associated with virtual nodes which does not act as collocation points in DiBFM [1,2]. Thus, only the variables associated with the source nodes form the unknowns in the final system equations and the system matrix in the DiBFM is of the same size as the conventional BEM with the source nodes alone. Due to a significant improvement in interpolation accuracy, the DiBFM can achieve higher computational accuracy. Furthermore, the second-layer interpolation is of the ability to approximate both 
continuous and discontinuous fields accurately. Therefore, the continuous displacement and discontinues tractions at the corners on the boudary are easily to be treated.

The boundary face method (BFM) proposed in [11] is also based on the boundary integral equation without geometric errors due to BFM uses CAD geometries directly in its implementation. In addition, the boundary face method inherits the advantages of BEM including dimension reduction, higher accuracy and infinite domain problems without domain truncation or artificial boundary conditions [12]. These advantages are also inherited by DiBFM.

Due to the above advantages, DiBFM has been applied to various 2D problems [1-5]. However, the $2 \mathrm{D}$ model is a simplification from $3 \mathrm{D}$ problem and it whichis difficult to truly reflect the 3D practical problem. Meanwhile, not all of the 3D practical models can be simplified to 2D models. In order to take full advantages in solving practical problems, this paper firstly extends the DiBFM to 3D elasticity problem with the dual interpolation element. For 3D elasticity problem, a general formulation of the DiBFM is also developed in detail.

The rest of the paper is organized as follows. The parameter mapping process for $3 \mathrm{D}$ problems is described in Section 2. Then the dual interpolation method for 3D problem is introduced in Section 3, and a general formulation of the problems is presented in Section 4. Numerical examples are presented in Section 5. Finally, the conclusions and future work are given in Section 6.

\section{Parameter mapping}

In DiBFM, the parameter mapping technique of the boundary faces is employed without geometric errors. The parameter mapping technique is a geometric mapping from the parametric space to physical space. It is well known that a surface in physical space can be exactly represented in parametric form as[13,14]:

$$
\boldsymbol{r}=\boldsymbol{r}(u, y)=\left(\begin{array}{ll}
(x & u
\end{array}\right) v,\left(\begin{array}{ll}
y
\end{array}\right) u(y \quad), u, v, \quad \in \quad u[0, \notin]
$$

where $\boldsymbol{r}$ is the position vector, $(u, v)$ is the parametric coordinate of the surface, and $(x, y, z)$ is the corresponding physical coordinate. The parameter mapping for a surface is shown in Fig.2. 


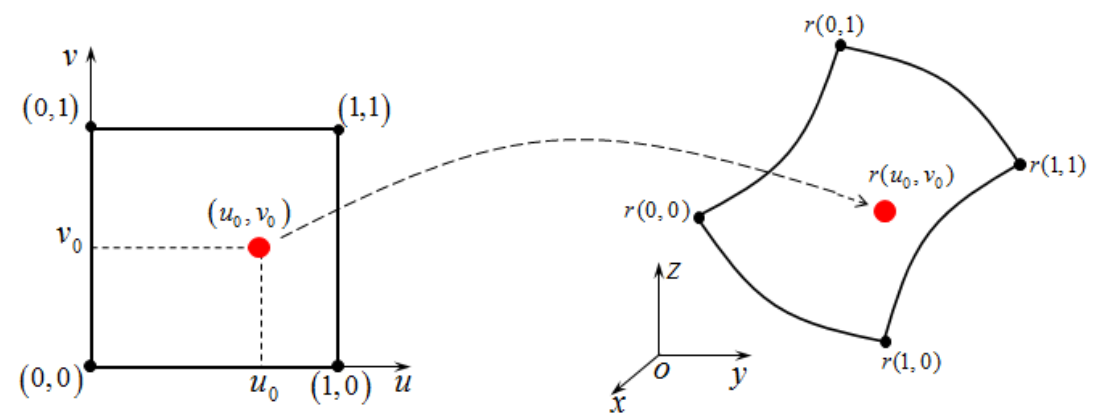

Fig.2. Mapping from the parametric space to the physical space.

In DiBFM, the integrand quantities, such as the coordinates of Gauss integration points, out normal and Jacobian, are calculated directly from the boundary faces, rather than from mesh elements [11]. It means that the coordinates of the Gaussian integration point are first represented by parameter coordinates. Then by the parameter mapping, the physical coordinates of Gauss integration points are specified. In this way, the physical coordinate will be located on the boundary faces (i.e. geometric surface), and thus no geometric errors occurs in its implementation (此段没有 表示太明白）. Similarly, the out normal and Jacobian can be obtained directly from boundary faces. More details can refer to the references $[11,13]$.

\section{Dual interpolation method in the DiBFM}

(此段可不要)

\subsection{The element in DiBFM}

In the DiBFM, the elements, called as dual interpolation elements, consist of source and virtual nodes as shown in Fig. 3 and Fig. 4 for triangular and quadrilateral elements.

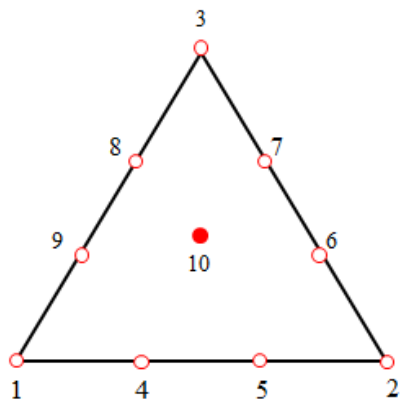

(a)

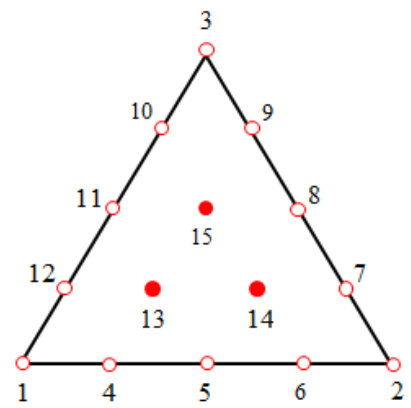

(b)

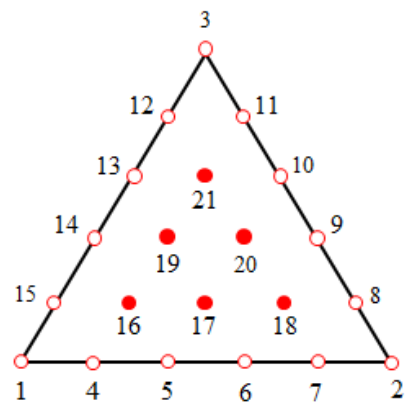

(c)

○ virtual node

- source node

Fig. 3. Dual interpolation elements for triangle: (a) TS1, (b) TS3, and (c) TS6. 


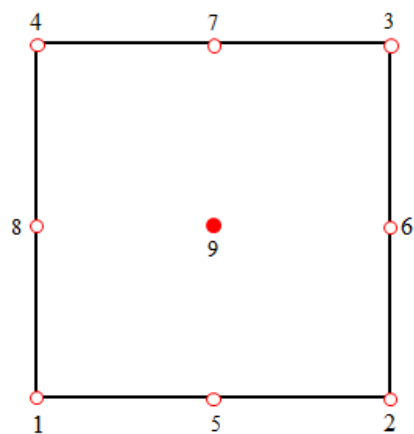

(a)

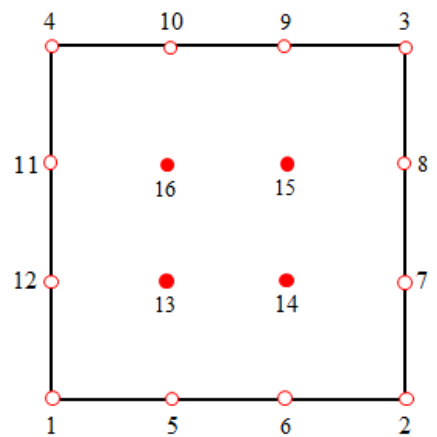

(b)

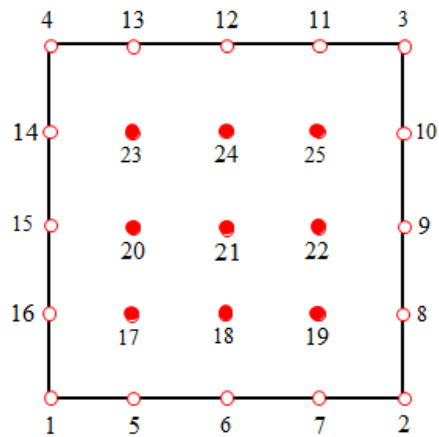

(c)

- virtual node

- source node

Fig. 4. Dual interpolation elements for quadrilateral: (a) QS1, (b) QS4, and (c) QS9.

As shown in Fig.3 and Fig.4, all nodes in a dual interpolation element are classified into two groups: in the first group, allsource nodes are located inside the element, and in the second group all virtual nodes are located on the edges and vertexes of the element. If the virtual nodes are removed, the dual interpolation element becomes a conventional discontinuous element. In this manner, the continuous and discontinuous element schemes can be unified by the dual interpolation element.

In the Fig.3, the elements are identified with the notation TS1, TS3 and TS6; while in Fig.4 the elements are identified with the notation QS1, QS4 and QS9, where T denotes the triangular element and Q denotes the quadrilateral element. The S indicates the source node in the continuous element. This notation is based on the fact that the degrees of freedom associated with the virtual nodes are eliminated, so that they do not appear in the final system equations in the DIBFM.

Compared with the interpolation function order of the conventional discontinuous elements which use the source nodes only, the addition of virtual nodes in dual interpolation elements signifies that the interpolation order in the dual interpolation elements is increased. For 2D problems, the interpolation order in the dual interpolation elements increases withtwo orders, which results in a significant improvement[1]. It is clear that this feature can be achieved to 3D problems.

\subsection{The first-layer interpolation}

The first-layer interpolation is similar to the interpolation in a conventional continuous boundary element. That is to say, both source nodes and virtual nodes are taken into account in dual interpolation elements. Thus, for an arbitrary quantity $\varphi$, the interpolation form is:

$$
\varphi(\xi, \eta)=\sum_{\alpha=1}^{n \alpha} N_{\alpha}^{s}(\xi, \eta) \varphi\left(Q_{\alpha}^{s}\right)+\sum_{\beta=1}^{n \beta} N_{\beta}^{v}(\xi, \eta) \varphi\left(Q_{\beta}^{v}\right)
$$


where $N_{\alpha}^{s}(\xi, \eta)$ and $N_{\beta}^{v}(\xi, \eta)$ denote the shape functions of the source node $\alpha$ and the virtual node $\beta \varphi\left(Q_{\alpha}^{s}\right)$ and $\varphi\left(Q_{\beta}^{v}\right)$ are the nodal values, $n \alpha$ and $n \beta$ (建议改成 $n_{s}$ 和 $n_{v}$ ) denote the total numbers of source nodes and virtual nodes in the dual interpolation element respectively. Normalized coordinate $(\xi, \eta)$ is used in the element, and $\xi, \eta \in[-1,1]$. In DiBFM, the virtual nodal value $\varphi\left(Q_{\beta}^{v}\right)$ is not independent which depends on the value of $\varphi$ at the source nodes to be determined by the second-layer interpolation.

It is noted that the shape functions $N_{\alpha}^{s}(\xi, \eta)$ and $N_{\beta}^{v}(\xi, \eta)$ in Eq. (2) are the Lagrange polynomials used in a conventional element with a number of nodes $(n \alpha+n \beta)$ (建议改成 $n_{s}$ 和 $n_{v}$ ). The derivation of the shape functions of the above elements can be referred to the work [15]. Here, only the shape functions are given.
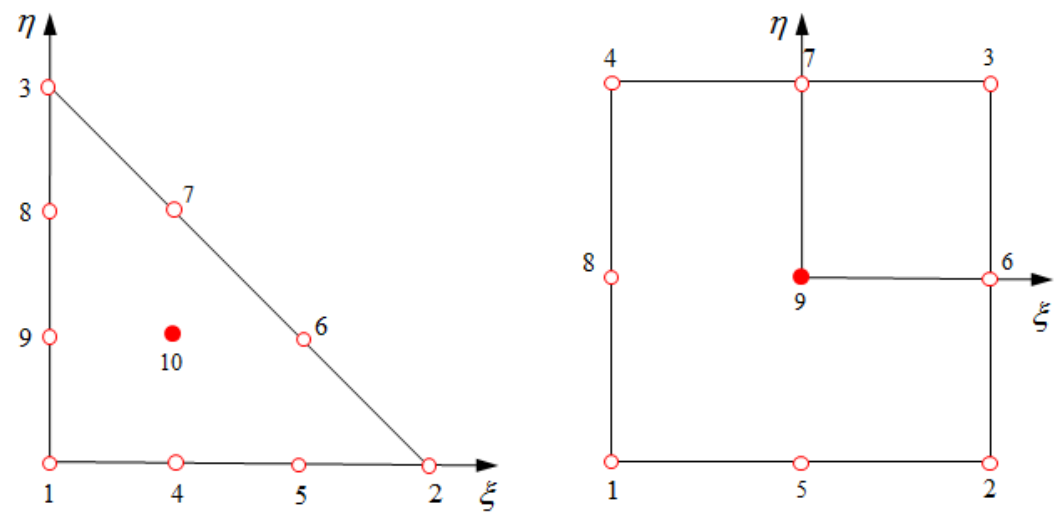

Fig. 5. Local parametric coordinate in element: TS1 and QS1.

$$
\begin{cases}N_{1}=0.5(3 \xi-1)(3 \xi-2) \xi & N_{6}=4.5 \eta(1-\xi-\eta)(3 \eta-1) \\ N_{2}=0.5(3 \eta-1)(3 \eta-2) \eta & N_{7}=4.5 \eta(1-\xi-\eta)(2-3 \xi-3 \eta) \\ N_{3}=0.5(2-3 \xi-3 \eta)(1-3 \xi-3 \eta)(1-\xi-\eta) & N_{8}=4.5 \xi(1-\xi-\eta)(2-3 \xi-3 \eta) \\ N_{4}=4.5 \xi \eta(3 \xi-1) & N_{9}=4.5 \xi(1-\xi-\eta)(3 \xi-1) \\ N_{5}=4.5 \xi \eta(3 \eta-1) & N_{10}=27 \xi \eta(1-\xi-\eta)\end{cases}
$$

(3)presented in Fig. 3 (a) and 


$$
\begin{cases}N_{1}=0.25 \xi \eta(\xi-1)(\eta-1) & N_{5}=0.5 \eta\left(1-\xi^{2}\right)(\eta-1) \\ N_{2}=0.25 \xi \eta(\xi+1)(\eta-1) & N_{6}=0.5 \xi(\xi+1)\left(1-\eta^{2}\right) \\ N_{3}=0.25 \xi \eta(\xi+1)(\eta+1) & N_{7}=0.5 \eta\left(1-\xi^{2}\right)(\eta+1) \\ N_{4}=0.25 \xi \eta(\xi-1)(\eta+1) & N_{8}=0.5 \xi(\xi-1)\left(1-\eta^{2}\right) \\ N_{9}=\left(1-\xi^{2}\right)\left(1-\eta^{2}\right) & \end{cases}
$$

in Fig. 4(a) respectively.

\subsection{The second-layer interpolation}

Same as in 2D problems [1,2], the second-layer interpolation is just utilized to construct the relationships between source and virtual nodes rather than the shape function of each Gauss point in the boundary integration. These relationships are employed to condense the degrees of freedom associated with virtual nodes. Thus, only the degrees of freedom associated with the source nodes form the unknowns of the final system equations in the DiBFM.

In this paper, the second-layer interpolation is constructed by the moving least square (MLS) approximation since the second-layer interpolation is able to approximate both continuous and discontinuous fields. It is obviously that it is effective to treat the continuous displacement and the discontinuous traction problems at the geometric edges andcorners.

\subsubsection{The moving least square approximation}

The approximation with MLS approximation is applied to construct the second-layer interpolation in this paper. The virtual nodal value isapproximated by:

$$
\varphi\left(Q_{\beta}^{v}\right)=\sum_{m=1}^{M} \psi_{m}^{v s}\left(u_{\beta}^{v}, v_{\beta}^{v}\right) \varphi\left(Q_{m}^{s}\right)
$$

where $M$ denotes the number of source nodes $Q_{m}^{s}$ collocated in the influence domain of virtual node $Q_{\beta}^{v}, \psi_{m}^{v s}\left(u_{\beta}^{v}, v_{\beta}^{v}\right)$ denotes the shape function of second-layer interpolation corresponding to source node $Q_{m}^{s}$ and $u_{\beta}^{v}$ and $v_{\beta}^{v}$ are the parametric coordinate of the virtual node $Q_{\beta}^{v}$, shown in Section 2 (坐标用 $\mathrm{u}, \mathrm{v}$ ? ) . The derivation of the Eq.(5) can be seen in the work [16]. 


\subsubsection{Approximation of continuous and discontinuous fields}

The continuous or discontinuous behavior at an element vertex can be accommodated by placing one or more virtual nodes at the vertex simply. Only one virtual node is needed to modela continuous field, and more than one virtual node are needed to approximate the discontinuous fields. If there are continuous and discontinuous fields, the number of the virtual nodes is equal to the number of the discontinuous field at the place. This feature is illustrated in Fig.6..

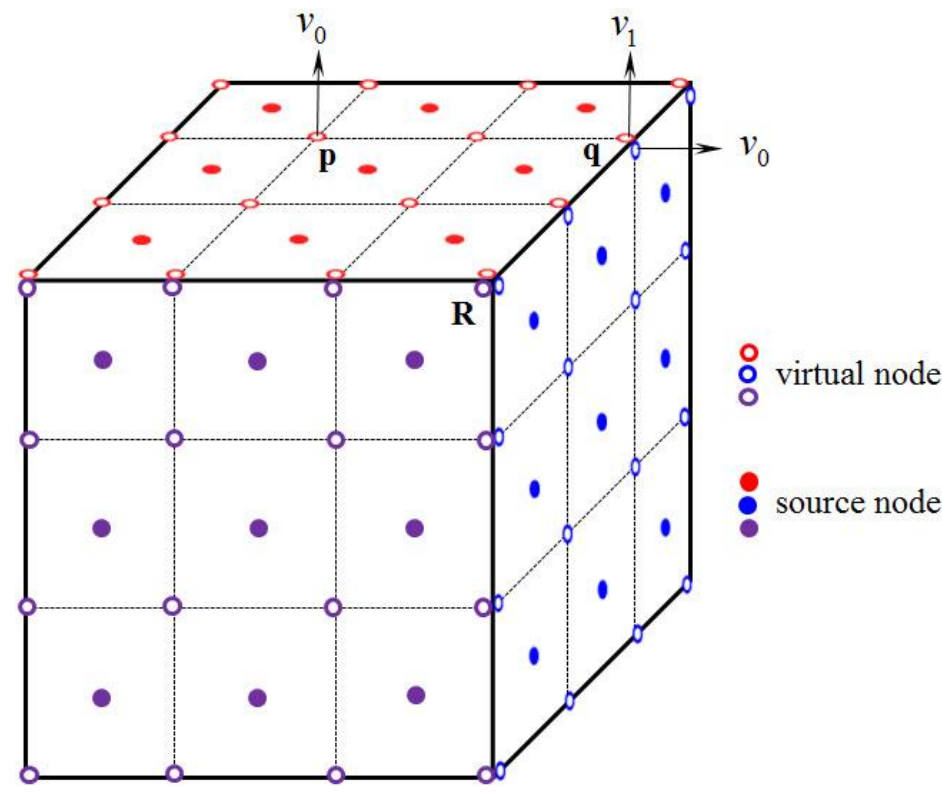

Fig. 6. Approximation of continuous and discontinuous fields

To model discontinuous fields, more than one virtual node need to be placed on the discontinuous boundary as shown in Fig.6. It well known that the traction field at either the geometric edges or the corners is discontinuous. Thus, two virtual nodes, $v_{0}$ and $v_{1}$, are considered at the geometric edges at point $\mathbf{q}$ shown in Fig.6. However, the influence domains of this two virtual nodes are different. As shown in Fig.6, the influence domain of $v_{0}$ covers nine source nodes in the blue, while the influence domain of $v_{1}$ covers the nine source nodes in the red. Since the influence domains are different, , the node values at $v_{0}$ and $v_{1}$ are different in the second-layer interpolation Eq.(5) in order to construct the discontinuous. By manipulating the influence domains of the three virtual nodes at the geometric corner (point $\mathbf{R}$ ), the traction discontinuous at the geometric corner is allowed.

For continuous fields, only a single virtual node, $v_{0}$, is placed at the element vertex at point $\mathbf{p}$ shown in Fig.6. For the second-layer interpolation in Eq.(5), the influence domain of this virtual 
node spans four elements. Since the virtual node $v_{0}$ is shared by the four elements, a continuity at the vertex is achieved.

Seeing from Fig.6, it is clear that the number of the virtual nodes at the geometric edges or corners is more than one due to the discontinuous traction condition at these points. The number of the virtual nodes is equal to the number of the discontinuous field.

Considering the continuous displacement condition at the geometric edges or corners, the influence domains of the virtual nodes at these locations should be the same. For example, for virtual nodes on the geometric edges, $v_{0}$ and $v_{1}$, the influence domain covers the nine source nodes in blue and the nine source nodes in red as well. Using the second-layer interpolation, the virtual nodal value is equal at $v_{0}$ and $v_{1}$ in order to achieve the continuous displacement Considering the continuous displacement condition and discontinuous traction condition at the geometric edges or corners need to be satisfied, the influence domains of the virtual nodes in displacement and traction are different. Thus, the continuous displacement and the discontinuous traction field at the geometric edges or corners can be dealt with easily.i.e. both continuous and discontinuous fields can be approximated naturally and accurately via manipulating the influence domain of virtual node in the second-layer interpolation $[1,2,4]$.

\section{DiBFM for elasticity problem}

\subsection{Boundary integral equation}

Consider an elasticity body occupying domain $\Omega \subset \square^{3}$ with a boundary $\Gamma$, an elastic solid without body forces, the boundary integral equation (BIE) [17-24] can be written as:

$$
c_{i j}(P) u_{j}(P)=\int_{\Gamma} U_{i j}(P, Q) t_{j}(Q) d \Gamma(Q)-\int_{\Gamma} T_{i j}(P, Q) u_{j}(Q) d \Gamma(Q), \quad P, Q \in \Gamma
$$

where $u_{j}$ is displacement; $t_{j}$ is traction and $i, j=(1,2,3)$. The $c_{i j}(P)$ denotes the coefficient matrix. The $U_{i j}(P, Q)$ and $T_{i j}(P, Q)$ are expressed as follow:

$$
\begin{gathered}
U_{i j}(P, Q)=\frac{1}{16 \pi G(1-v) r}\left[(3-4 v) \delta_{i j}+r_{, i} r_{, j}\right] \\
T_{i j}(P, Q)=\frac{1}{8 \pi(1-v) r^{2}}\left\{\frac{\partial r}{\partial n}\left[(1-2 v) \delta_{i j}+3 r_{, i} r_{, j}\right]+(1-2 v)\left(n_{j} r_{, i}-n_{i} r_{, j}\right)\right\}
\end{gathered}
$$


where $r$ denotes the distance between the source node $P$ and the field point $Q$, and $n$ is the unit outward normal at the point $Q, v$ denotes the Poisson's ratio and $G$ the shear modulus.

\subsection{Discretization of the BIE for elasticity problems}

In the DiBFM, the BIE is discretized by dual interpolation elements with the source nodes $P_{k}$ $(k=1,2, \ldots, \mathrm{NS})$ are considered only, where NS is the total number of the source nodes. After discretizing, the BIE for elasticity problems yields:

$$
\sum_{e=1}^{n e}\left[\sum_{\alpha=1}^{n \alpha} h_{i j}^{s s}\left(P_{k}\right) u_{j}\left(Q_{e(a)}^{s}\right)+\sum_{\beta=1}^{n \beta} h_{i j}^{s v}\left(P_{k}\right) u_{j}\left(Q_{e(\beta)}^{v}\right)\right]=\sum_{e=1}^{n e}\left[\sum_{\alpha=1}^{n \alpha} g_{i j}^{s s}\left(P_{k}\right) t_{j}\left(Q_{e(a)}^{s}\right)+\sum_{\beta=1}^{n \beta} g_{i j}^{s v}\left(P_{k}\right) t_{j}\left(Q_{e(\beta)}^{v}\right)\right]
$$

in which

$$
\begin{aligned}
& h_{i j}^{s s}\left(P_{k}\right)=\int_{\Gamma_{e}} T_{i}\left(P_{*} Q\right) N_{(\alpha) e}(\Phi) d+\underset{2}{\operatorname{\theta }} \not \delta \delta_{\alpha i j}^{k} \\
& h_{i j}^{s v}\left(P_{k}\right)=\int_{\Gamma_{e}} T_{i j}\left(P_{k}, Q\right) N_{e(\beta)}^{v}(Q) d \Gamma(Q) \\
& g_{i j}^{s s}\left(P_{k}\right)=\int_{\Gamma_{e}} U_{i j}\left(P_{k}, Q\right) N_{e(\alpha)}^{s}(Q) d \Gamma(Q) \\
& g_{i j}^{s v}\left(P_{k}\right)=\int_{\Gamma_{e}} U_{i j}\left(P_{k}, Q\right) N_{e(\beta)}^{v}(Q) d \Gamma(Q)
\end{aligned}
$$

and

$$
\delta_{e(\alpha)}^{k}=\left\{\begin{array}{l}
1, \text { if source node } P_{k} \text { is the } \alpha^{\text {th }} \text { source node in the } e^{\text {th }} \text { element } \\
0,
\end{array}\right.
$$

where $\Gamma_{e}$ denotes the boundary $e$-th element, $N_{e(\alpha)}^{s}, t_{j}\left(Q_{e(a)}^{s}\right)$ and $u_{j}\left(Q_{e(a)}^{s}\right)$ denote the shape function, nodal values of the traction and displacement of the $\alpha$-th source node in the e-th element, $N_{e(\beta)}^{v}, t_{j}\left(Q_{e(\beta)}^{v}\right)$ and $u_{j}\left(Q_{e(\beta)}^{v}\right)$ are shape function, nodal value of the traction and displacement of the $\beta$-th virtual node in the $e^{\text {th }}$ element, respectively.

Eq. (9) can be arranged in a matrix form as:

$$
\sum_{j=1}^{3}\left(\left[\begin{array}{l}
\mathbf{H}_{1 j}^{s s} \\
\mathbf{H}_{2 j}^{s s} \\
\mathbf{H}_{3 j}^{s s}
\end{array}\right]\left\{\mathbf{u}_{j}^{s}\right\}+\left[\begin{array}{l}
\mathbf{H}_{1 j}^{s v} \\
\mathbf{H}_{2 j}^{s v} \\
\mathbf{H}_{3 j}^{s v}
\end{array}\right]\left\{\mathbf{u}_{j}^{v}\right\}\right)=\sum_{j=1}^{3}\left(\left[\begin{array}{l}
\mathbf{G}_{1 j}^{s s} \\
\mathbf{G}_{2 j}^{s s} \\
\mathbf{G}_{3 j}^{s s}
\end{array}\right]\left\{\mathbf{t}_{j}^{s}\right\}+\left[\begin{array}{c}
\mathbf{G}_{1 j}^{s v} \\
\mathbf{G}_{2 j}^{s v} \\
\mathbf{G}_{3 j}^{s v}
\end{array}\right]\left\{\mathbf{t}_{j}^{v}\right\}\right)
$$

where $\mathbf{u}_{j}^{s}$ and $\mathbf{t}_{j}^{s}$ denote the vectors of displacement and traction for all source nodes, $\mathbf{u}_{j}^{v}$ and $\mathbf{t}_{j}^{v}$ aredisplacement and traction vectors for all virtual nodes, $\mathbf{H}_{i j}^{s s}, \mathbf{G}_{i j}^{s s}, \mathbf{H}_{i j}^{s v}$ and $\mathbf{G}_{i j}^{s v}$ are the 
coefficient matrices corresponding to vectors $\mathbf{u}_{j}^{s}, \mathbf{t}_{j}^{s}, \mathbf{u}_{j}^{v}$ and $\mathbf{t}_{j}^{v}$, respectively. Considering the boundary conditions, we can rewrite Eq.(10) as:

$$
\sum_{j=1}^{3}\left(\left[\begin{array}{cc}
\mathbf{H}_{1 d_{j}}^{s s} & \mathbf{H}_{1 n_{j}}^{s s} \\
\mathbf{H}_{2 d_{j}}^{s s} & \mathbf{H}_{2 n_{j}}^{s s} \\
\mathbf{H}_{3 d_{j}}^{s s} & \mathbf{H}_{3 n_{j}}^{s s}
\end{array}\right]\left\{\begin{array}{l}
\overline{\mathbf{u}}_{d_{j}}^{s} \\
\mathbf{u}_{n_{j}}^{s}
\end{array}\right\}+\left[\begin{array}{cc}
\mathbf{H}_{1 d_{j}}^{s v} & \mathbf{H}_{1 n_{j}}^{s v} \\
\mathbf{H}_{2 d_{j}}^{s v} & \mathbf{H}_{2 n_{j}}^{s v} \\
\mathbf{H}_{3 d_{j}}^{s v} & \mathbf{H}_{3 n_{j}}^{s v}
\end{array}\right]\left\{\begin{array}{l}
\overline{\mathbf{u}}_{d_{j}}^{v} \\
\mathbf{u}_{n_{j}}^{v}
\end{array}\right\}\right)=\sum_{j=1}^{3}\left(\left[\begin{array}{cc}
\mathbf{G}_{1 d_{j}}^{s s} & \mathbf{G}_{1 n_{j}}^{s s} \\
\mathbf{G}_{2 d_{j}}^{s s} & \mathbf{G}_{2 n_{j}}^{s s} \\
\mathbf{G}_{3 d_{j}}^{s s} & \mathbf{G}_{3 n_{j}}^{s s}
\end{array}\right]\left\{\begin{array}{l}
\mathbf{t}_{d_{j}}^{s} \\
\overline{\mathbf{t}}_{n_{j}}^{s}
\end{array}\right\}+\left[\begin{array}{cc}
\mathbf{G}_{1 d_{j}}^{s v} & \mathbf{G}_{1 n_{j}}^{s v} \\
\mathbf{G}_{2 d_{j}}^{s v} & \mathbf{G}_{2 n_{j}}^{s v} \\
\mathbf{G}_{3 d_{j}}^{s v} & \mathbf{G}_{3 n_{j}}^{s v}
\end{array}\right]\left\{\begin{array}{l}
\mathbf{t}_{d_{j}}^{v} \\
\overline{\mathbf{t}}_{n_{j}}^{v}
\end{array}\right\}\right)
$$

where the subscripts $d_{j}$ and $n_{j}$ indicate the Dirichlet (displacement) and Neumann (traction) boundary conditions in the $j$ direction, $\overline{\mathbf{u}}_{d_{j}}^{s}, \overline{\mathbf{u}}_{d_{j}}^{v}$ andand $\overline{\mathbf{t}}_{n_{j}}^{s}, \overline{\mathbf{t}}_{n_{j}}^{v}$ are vectors of the displacement and the traction of the source node and the virtual node specified in the $j$ direction respectively.

\subsection{Condensation the degrees of freedom of the virtual nodes}

As presented in Section 3.1, the BIEs are only collocated at source nodes. Thus, the number of linear equations is less than that of unknown nodal values inEq. (11). In order to make the final equations solvable, additional constraint equations are needed to condense the degrees of freedom of the virtual nodes. The additional constraint equations are provided by the second-layer interpolation as:

$$
\begin{aligned}
& \left\{\mathbf{u}_{n_{j}}^{v}\right\}=\boldsymbol{\Psi}_{n_{j} n_{j}}^{v s}\left\{\mathbf{u}_{n_{j}}^{s}\right\}, j=(1,2,3) \\
& \left\{\mathbf{t}_{d_{j}}^{v}\right\}=\boldsymbol{\Psi}_{d_{j} d_{j}}^{v s}\{\mathbf{t}\}, j=(1,2)
\end{aligned}
$$

where $\boldsymbol{\Psi}_{n_{j} n_{j}}^{v s}$ and $\boldsymbol{\Theta}_{d_{j} d_{j}}^{v s}$ denote shape function matrices constructed by the second-layer interpolation, see the Section 3.3.1.

Substituting Eqs (12) and (13) into Eq. (11) yields:

$$
\sum_{j=1}^{3}\left(\left[\begin{array}{cc}
\mathbf{H}_{1 d_{j}}^{s s} & \overline{\mathbf{H}}_{1 n_{j}}^{s s} \\
\mathbf{H}_{2 d_{j}}^{s s} & \overline{\mathbf{H}}_{2 n_{j}}^{s s} \\
\mathbf{H}_{3 d_{j}}^{s s} & \overline{\mathbf{H}}_{3 n_{j}}^{s s}
\end{array}\right]\left\{\begin{array}{l}
\overline{\mathbf{u}}_{d_{j}}^{s} \\
\mathbf{u}_{n_{j}}^{s}
\end{array}\right\}+\left[\begin{array}{c}
\mathbf{H}_{1 d_{1}}^{s v} \\
\mathbf{H}_{2 d_{1}}^{s v} \\
\mathbf{H}_{3 d_{1}}^{s v}
\end{array}\right]\left\{\overline{\mathbf{u}}_{d_{j}}^{v}\right\}\right)=\sum_{j=1}^{3}\left(\left[\begin{array}{cc}
\overline{\mathbf{G}}_{1 d_{j}}^{s s} & \mathbf{G}_{1 n_{j}}^{s s} \\
\overline{\mathbf{G}}_{2 d_{j}}^{s s} & \mathbf{G}_{2 n_{j}}^{s s} \\
\overline{\mathbf{G}}_{3 d_{j}}^{s s} & \mathbf{G}_{3 n_{j}}^{s s}
\end{array}\right]\left\{\begin{array}{c}
\mathbf{t}_{d_{j}}^{s} \\
\overline{\mathbf{t}}_{n_{j}}^{s}
\end{array}\right\}+\left[\begin{array}{c}
\mathbf{G}_{1 n_{1}}^{s v} \\
\mathbf{G}_{2 n_{1}}^{s v} \\
\mathbf{G}_{3 n_{1}}^{s v}
\end{array}\right]\left\{\overline{\mathbf{t}}_{n_{j}}^{v}\right\}\right)
$$

in which 


$$
\begin{aligned}
& {\left[\begin{array}{c}
\overline{\mathbf{H}}_{1 n_{j}}^{s s} \\
\overline{\mathbf{H}}_{2 n_{j}}^{s s} \\
\overline{\mathbf{H}}_{3 n_{j}}^{s s}
\end{array}\right]=\left[\begin{array}{l}
\mathbf{H}_{1 n_{j}}^{s s} \\
\mathbf{H}_{2 n_{j}}^{s s} \\
\mathbf{H}_{3 n_{j}}^{s s}
\end{array}\right]+\left[\begin{array}{l}
\mathbf{H}_{1 n_{j}}^{s v} \\
\mathbf{H}_{2 n_{j}}^{s v} \\
\mathbf{H}_{3 n_{j}}^{s v}
\end{array}\right] \boldsymbol{\Psi}_{n_{j} n_{j}}^{c s}}
\end{aligned}
$$

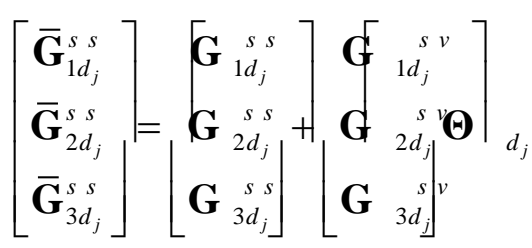

\subsection{Solution for elasticity problems}

Imposing the boundary conditions at each source and virtual node and exchanging the columns of matrices in Eq. (14), the final system equations can be rewritten as:

$$
\mathbf{A x}=\mathbf{b}
$$

in which

$$
\begin{aligned}
& \mathbf{A}=\left[\begin{array}{cccccc}
-\overline{\mathbf{G}}_{1 d_{1}}^{s s} & \overline{\mathbf{H}}_{1 n_{1}}^{s s} & -\overline{\mathbf{G}}_{1 d_{2}}^{s s} & \overline{\mathbf{H}}_{1 n_{2}}^{s s} & -\overline{\mathbf{G}}_{1 d_{3}}^{s s} & \overline{\mathbf{H}}_{1 n_{3}}^{s s} \\
-\overline{\mathbf{G}}_{2 d_{1}}^{s s} & \overline{\mathbf{H}}_{2 n_{1}}^{s s} & -\overline{\mathbf{G}}_{2 d_{2}}^{s s} & \overline{\mathbf{H}}_{2 n_{2}}^{s s} & -\overline{\mathbf{G}}_{2 d_{3}}^{s s} & \overline{\mathbf{H}}_{2 n_{3}}^{s s} \\
-\overline{\mathbf{G}}_{3 d_{1}}^{s s} & \overline{\mathbf{H}}_{3 n_{1}}^{s s} & -\overline{\mathbf{G}}_{3 d_{2}}^{s s} & \overline{\mathbf{H}}_{3 n_{2}}^{s s} & -\overline{\mathbf{G}}_{3 d_{3}}^{s s} & \overline{\mathbf{H}}_{3 n_{3}}^{s s}
\end{array}\right] \\
& \mathbf{x}=\left\{\begin{array}{llllll}
\mathbf{t}_{d_{1}}^{s} & \mathbf{u}_{n_{1}}^{s} & \mathbf{t}_{d_{2}}^{s} & \mathbf{u}_{n_{2}}^{s} & \mathbf{t}_{d_{3}}^{s} & \mathbf{u}_{n_{3}}^{s}
\end{array}\right\}^{\mathrm{T}} \\
& \mathbf{b}=\sum_{j=1}^{3}\left(\left[\begin{array}{cc}
-\mathbf{H}_{1 d_{j}}^{s s} & \mathbf{G}_{1 n_{j}}^{s s} \\
-\mathbf{H}_{2 d_{j}}^{s s} & \mathbf{G}_{2 n_{j}}^{s s} \\
-\mathbf{H}_{3 d_{j}}^{s s} & \mathbf{G}_{3 n_{j}}^{s s}
\end{array}\right]\left\{\begin{array}{c}
\overline{\mathbf{u}}_{d_{j}}^{s} \\
\overline{\mathbf{t}}_{n_{j}}^{s}
\end{array}\right\}+\left[\begin{array}{cc}
-\mathbf{H}_{1 d_{j}}^{s v} & \mathbf{G}_{1 n_{j}}^{s v} \\
-\mathbf{H}_{2 d_{j}}^{s v} & \mathbf{G}_{2 n_{j}}^{s v} \\
-\mathbf{H}_{3 d_{j}}^{s v} & \mathbf{G}_{3 n_{j}}^{s v}
\end{array}\right]\left\{\begin{array}{c}
\overline{\mathbf{u}}_{d_{j}}^{v} \\
\overline{\mathbf{t}}_{n_{j}}^{v}
\end{array}\right\}\right)
\end{aligned}
$$

For 3D elasticity problems, the size of matrix $\mathbf{A}$ is $3 \mathrm{NS}, \mathbf{x}$ is unknown at the source node and $\mathbf{b}$ is known with boundary conditions.

In this paper, the Gaussian elimination method [25] is used to obtain solutions in Eq. (15). By using the second-layer interpolation in Eq.(12) and Eq.(13), all nodal values at virtual nodes can be determined.

Due to the unknown vector of the final system equations involving the source node only, the size of the final system equations in the DiBFM is the same as that in the conventional BEM using source nodes alone (seeing from the Eq. (15)). As a result, the efficiency in DiBFM remains unchanged. 


\section{Numerical examples}

In this section, three examples are presented. The first example is used to demonstrate the accuracy and convergence of the DiBFM for solving 3D elastic problems. The other two examples demonstrate further the high accuracy of the proposed method for realistic engineering problems.

\subsection{Analytical displacement field problem}

To evaluate the degrees of accuracy and convergence usingthe DiBFM, an analytical displacement field problem for a cube as shown in Fig.7(a) is considered. The Young's modulus $E=1$ and Poisson's ratio $v=0.25$. The analytical displacement fields imposed on all faces are:

$$
\left\{\begin{array}{l}
u_{x}=\left(y^{3}-z^{3}\right)-3(y-z) x^{2} \\
u_{y}=\left(z^{3}-x^{3}\right)-3(z-x) y^{2} \\
u_{z}=\left(x^{3}-y^{3}\right)-3(x-y) z^{2}
\end{array}\right.
$$

（检查一下此解是否满足平衡方程）From the Eq.(16), it can be seen that the displacement field is a cubic function. Then, due to the differentiation operation, the stress field is a quadratic field function. The accuracy of the Von Mises stress will be used to study the accuracy and convergence. The accuracy is calculated by the following equation

$$
\text { error }=\frac{1}{|v|_{\text {max }}} \sqrt{\left.\frac{1}{M} \sum_{i=1}^{M}\left[v_{i}^{(e)}-v_{i}^{n(}\right]^{\prime}\right)}
$$

where $|v|_{\max }$ is the maximum Von Mises stress value (建议用 $\sigma_{\text {von }}$ ) on sample points, and the superscripts $(e)$ and $(n)$ refer to the exact and numerical solutions, respectively. The total number of sample points $\mathrm{M}$ is 180 , and 60 sample points for each diagonal surfaces shown in Fig.7. In this example, both element QS1(seeing Fig.3(a)) and TS1(seeing Fig.4(a)) are investigated.

The computational errors with different number of source nodes $(N S)$ are listed in Table 1. From Table 1 , the accuracy is $10^{-2}$ even if only 96 QS1 or 84 TS1 is used to approximate quadratic stress field, which demonstrates the degree of accuracy of the DiBFM. It can be observed that when the number of elements increases, the accuracy increases. This characteristic confirms the convergence of the DiBFM. 


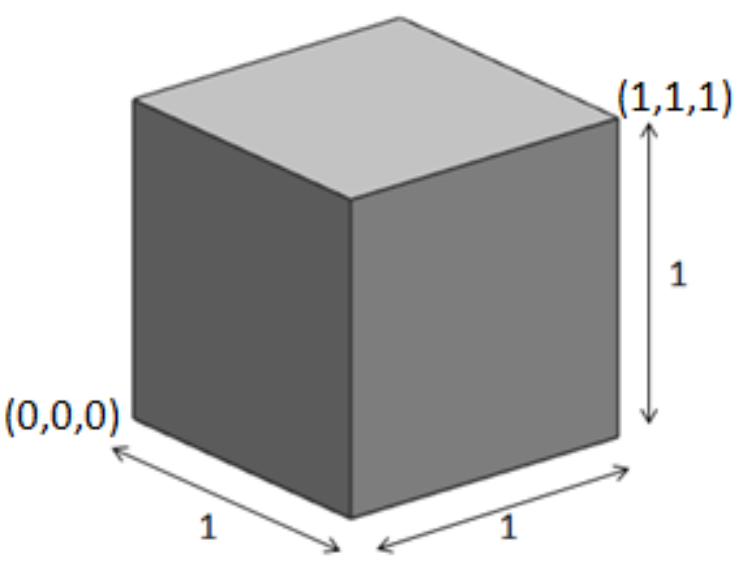

(a) Geometric model

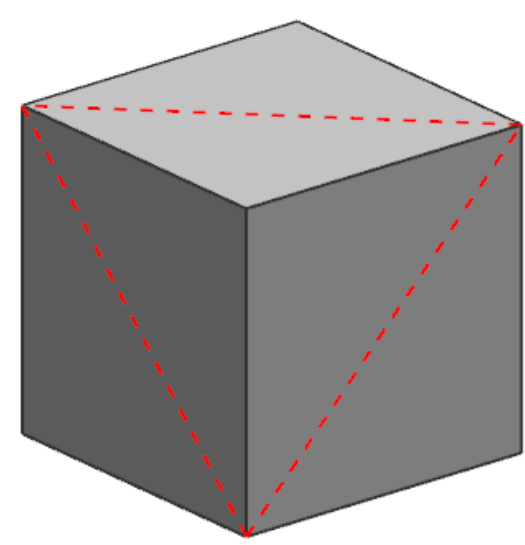

(b) The distribution of the sample points

Fig. 7. The analytical field problem.

Table 1. Comparison of convergence of the DiBFM with the dual interpolation elements

QS1 and TS1.

\begin{tabular}{ccccc}
\hline \multicolumn{2}{c}{ DiBFM QS1 } & & \multicolumn{2}{c}{ DiBFM TS1 } \\
\cline { 1 - 2 } \cline { 5 - 6 }$N S$ & Err_Von Mises & & $N S$ & Er__Von Mises \\
\hline 96 & $3.65 \mathrm{e}-04$ & & 84 & $1.29 \mathrm{e}-02$ \\
150 & $1.96 \mathrm{e}-04$ & & 132 & $5.00 \mathrm{e}-03$ \\
600 & $4.76 \mathrm{e}-05$ & & 606 & $9.20 \mathrm{e}-04$ \\
1,176 & $3.86 \mathrm{e}-05$ & & 1,284 & $3.57 \mathrm{e}-04$ \\
2,400 & $3.63 \mathrm{e}-05$ & & 2,430 & $1.09 \mathrm{e}-04$ \\
\hline
\end{tabular}

\subsection{Three-way pipe with a rounded corner}

In this example, a three-way pipe with a rounded corner is analyzed to show the capability of DiBFM to solve the problem of the structure as shown in Fig.8(a). The dimensions of the horizontal hollow cylinder are $\mathrm{R}=10, \mathrm{r}=6$ and $\mathrm{h}=15$ and the dimensions of the vertical hollow cylinder are $\mathrm{R}=6, \mathrm{r}=4$ and $\mathrm{h}=11$. In addition, the rounded corner between the two hollow cylinders is $r=0.4$. In this model, Young's modules $E=200,000$ and Poisson's ratio $v=0.25$. The boundary conditions are specified as: one side of the horizontal hollow cylinder is clamped, the top face of the vertical hollow cylinder is subjected to a uniform traction $(p=10)$ 【不用粗体】. In the DiBFM analysis, the total number of source nodes is 7527 and the element mesh is shown in Fig.8(b). The FEM is used to introduced as a reference with 1519450 quadratic tetrahedron elements.

We consider the Von Mises stress on the rounded corner. Since the fixed end is of a sharp corner, the Von Mises stress at this point is not convergent. , i.e.. when the mesh density increases, the Von 
Mises stress value at the corner increases. Thus, the Von Mises stress on the rounded corner is chosen for comparison. The displacement and Von Mises stress results are shown in Fig.9 and Fig.10 respectively.

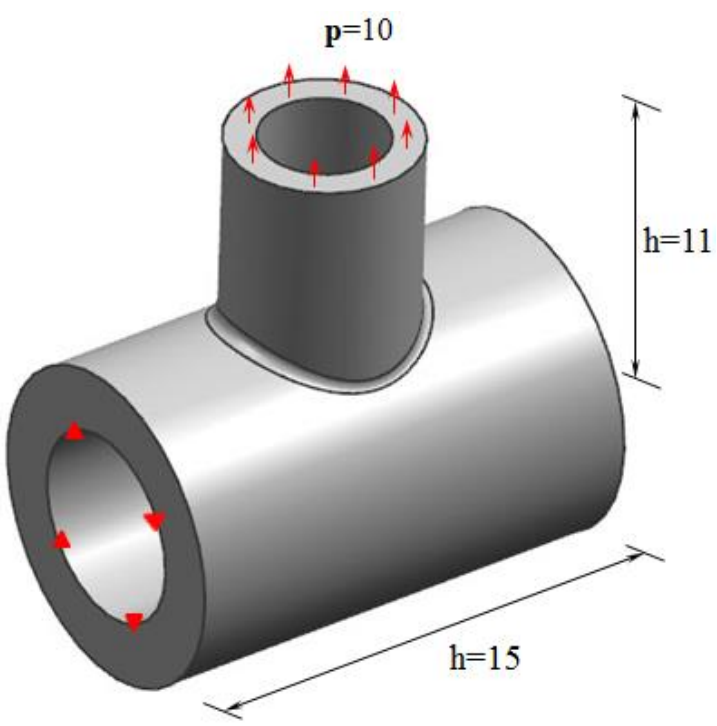

(a) Geometry and boundary conditions

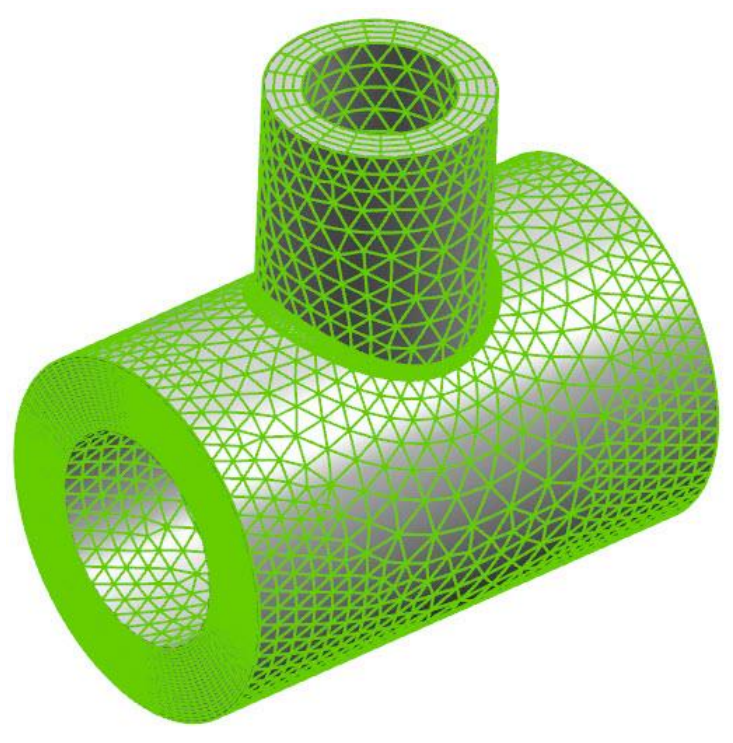

(b) Mesh used in DiBFM

Fig. 8. Three-way pipe

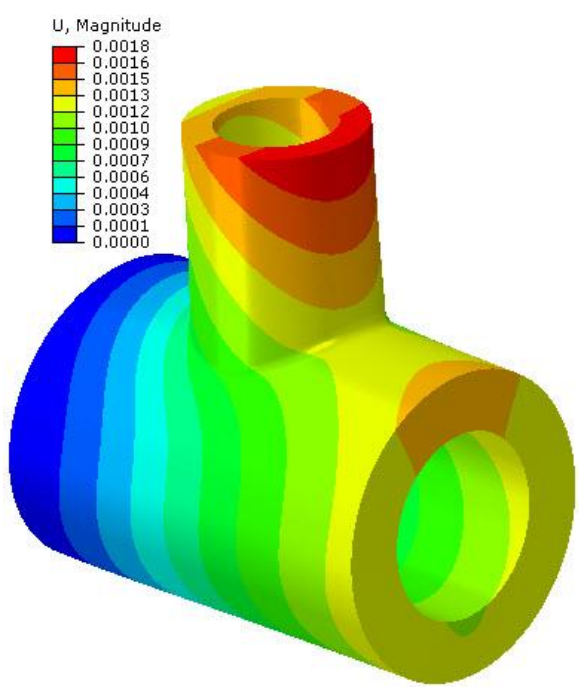

(a) FEM

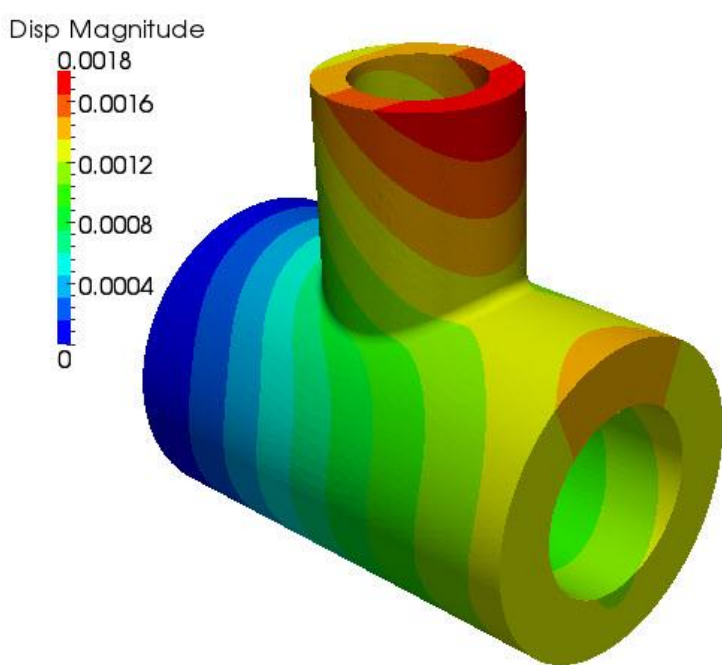

(b) DiBFM

Fig. 9. The displacement results of the two methods 


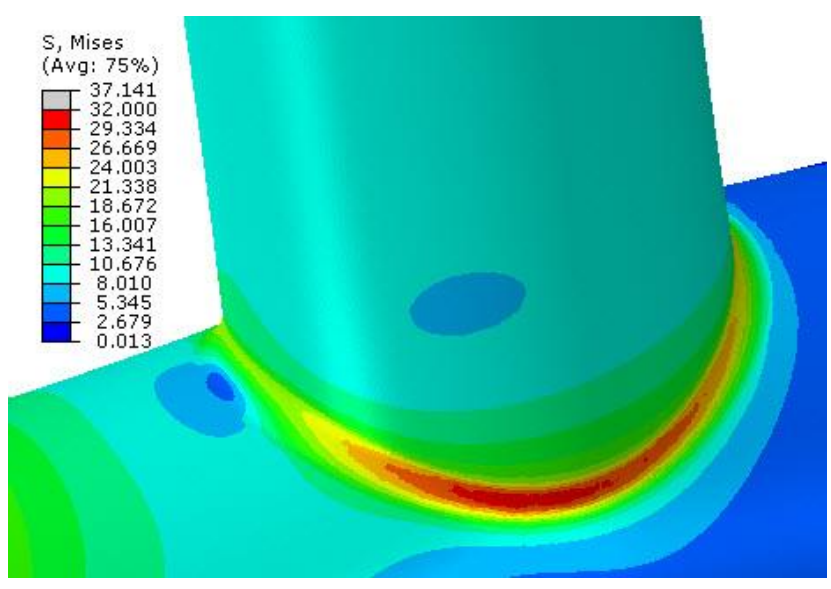

(a) FEM

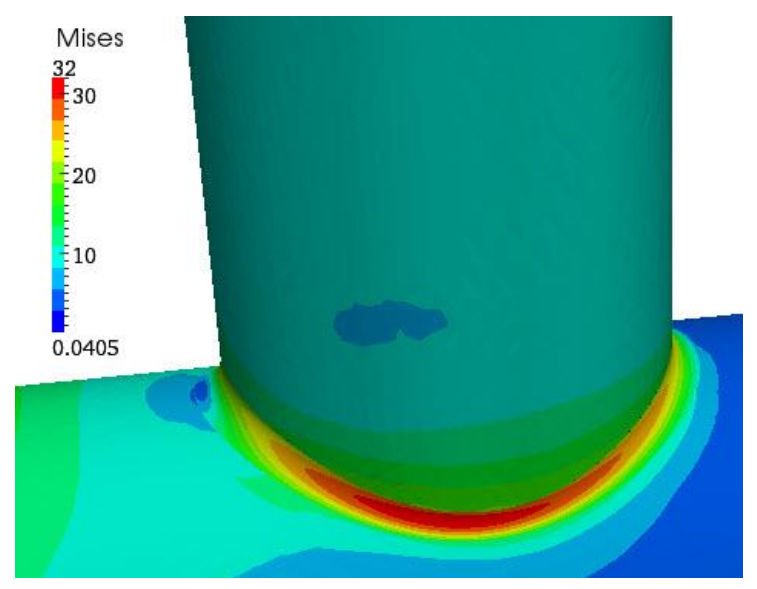

(b) DiBFM

Fig. 10. The Von Mises stress at the rounded corner in the two methods.

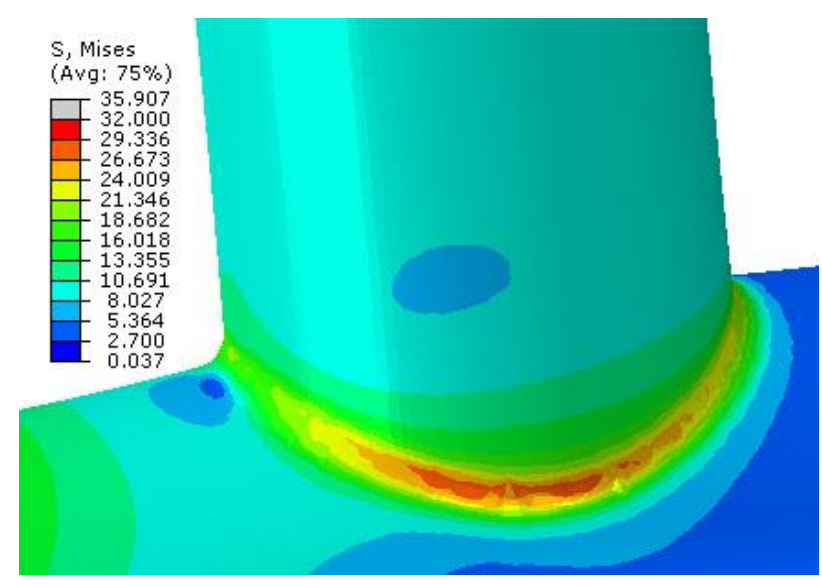

(a) Node number-493657

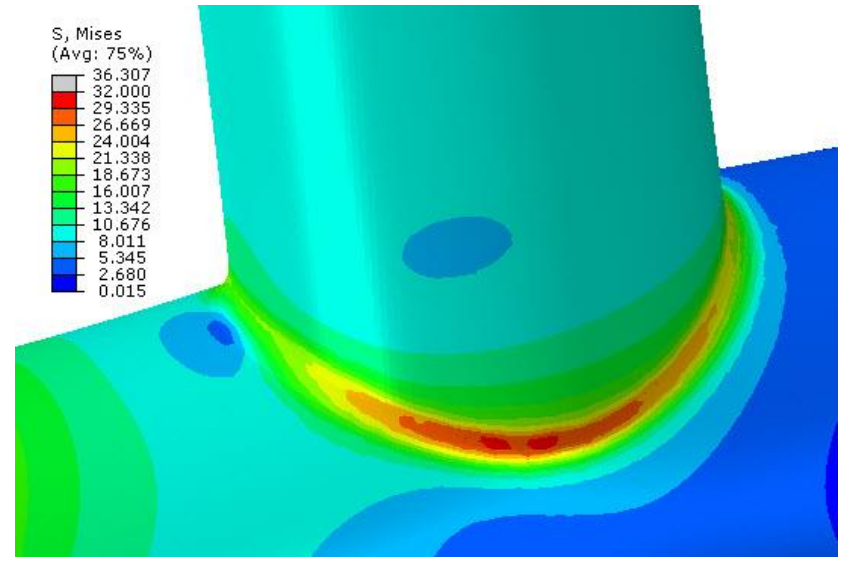

(b) Node number-928384

Fig. 11. The Von Mises stress at the rounded corner with different numbers of node in FEM

From the Fig.9, it can be seen that the displacements given by DiBFM are almost coincident with that by FEM. Fig.10 demonstrates the excellent agreements between these two methods with von Mises stress around the rounded corner. In Fig.11, the distribution of von Mises stress at the rounded corner is not smooth when using 493657 nodes and 928384 nodes in FEM 【这两句不是很 清楚, 要说明什么? 】. Besides, even if the total number of nodes is 1519450 , the stress distribution at the fillet obtained by FEM is less smooth than that obtained by DiBFM, seeing from Fig.10. However, the total number of source nodes in DiBFM is 7527 only. In Fig.10, the difference for von Mises stress on the cylinder surface is due to the fact that the meshes by two methods are different. In DiBFM, a coarse mesh is adopted at the cylinder surface, which results in an uneven contour of the von Mises stress. In other words, the smoother von Mises stress distribution in DiBFM illustrates that the DiBFM is more suitable for solving the structure with small features. 


\subsection{Angle steel}

An angle steel model is analyzed in this example to show the capability of the DiBFM to solve the practical problems in engineering. The geometric model is shown in Fig.12(a). In this model, Young's modules E=20000 and Poisson's ratio $v=0.3$. The bottom of the angle steel is fixed, and the back of the angle steel is subjected to a uniform pressure $\mathrm{p}=1.0$. In DiBFM, the total number of source nodes is 6937, and the mesh can be seen in Fig.12(b). In FEM, the quadratic tetrahedron elements with 1519450 nodes are used. The displacement and von Mises stress results are shown in Fig.13, Fig.14 and the maximum displacement and von Mises stress are presented in Table 2.

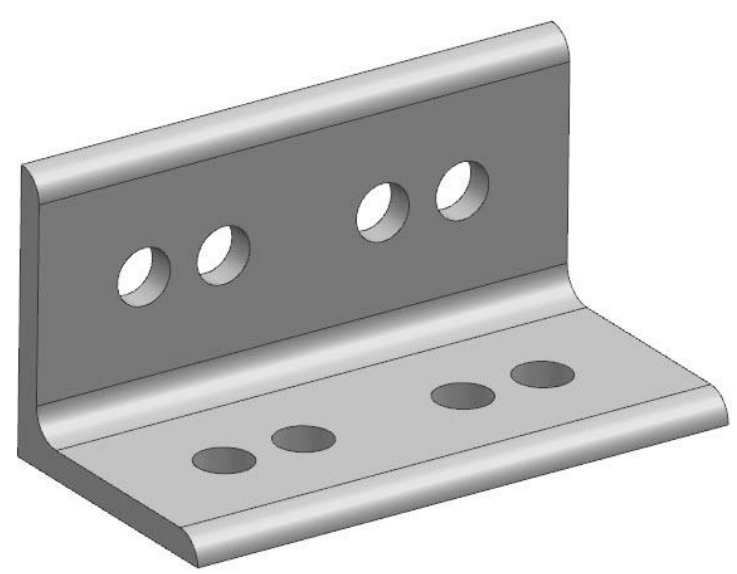

(a) Geometric model

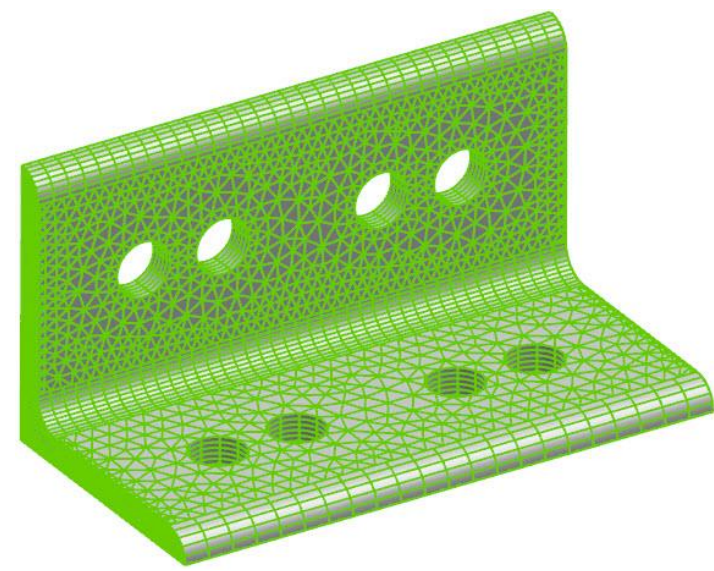

(b) Mesh in DiBFM

Fig. 12. Angle steel

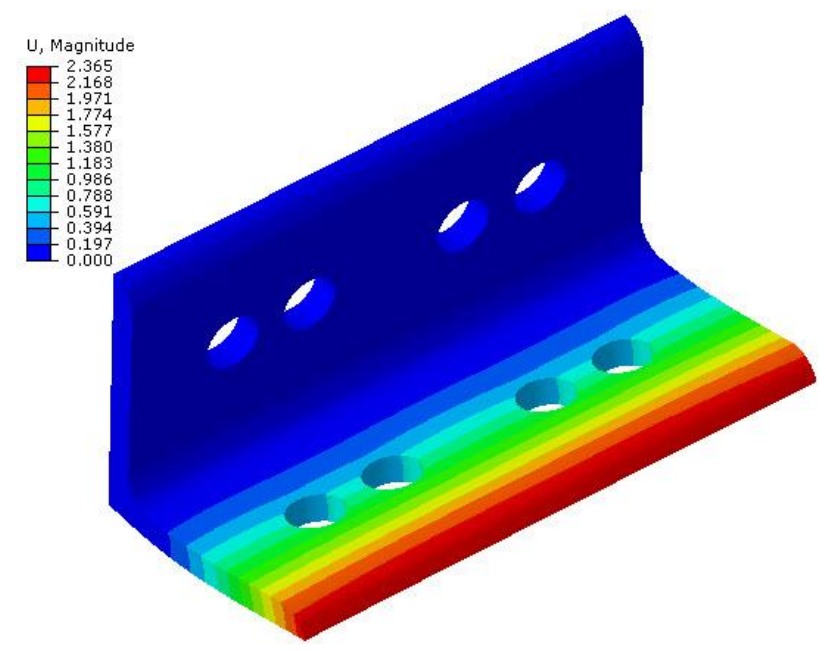

(a) FEM

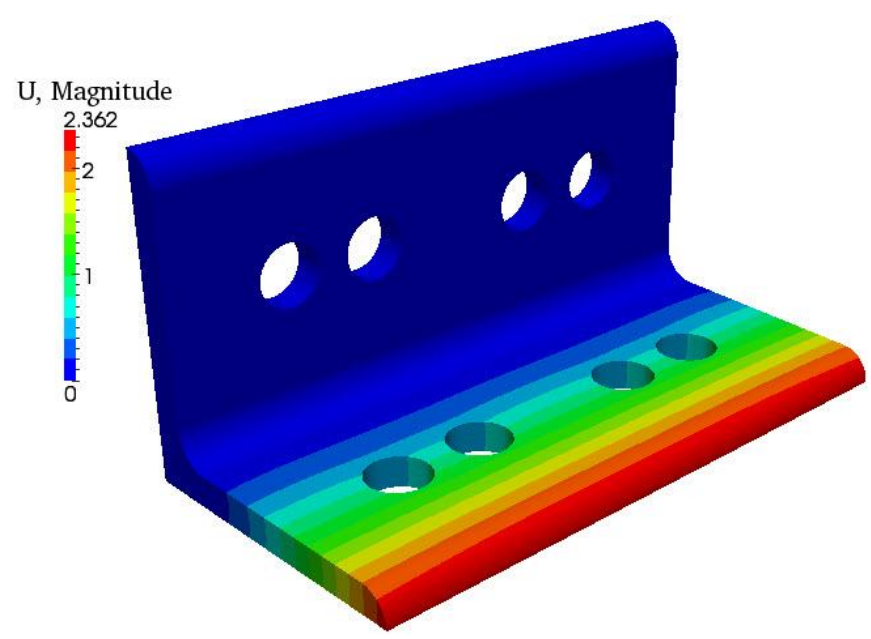

(b) DiBFM

Fig. 13. The displacement results by the two methods 


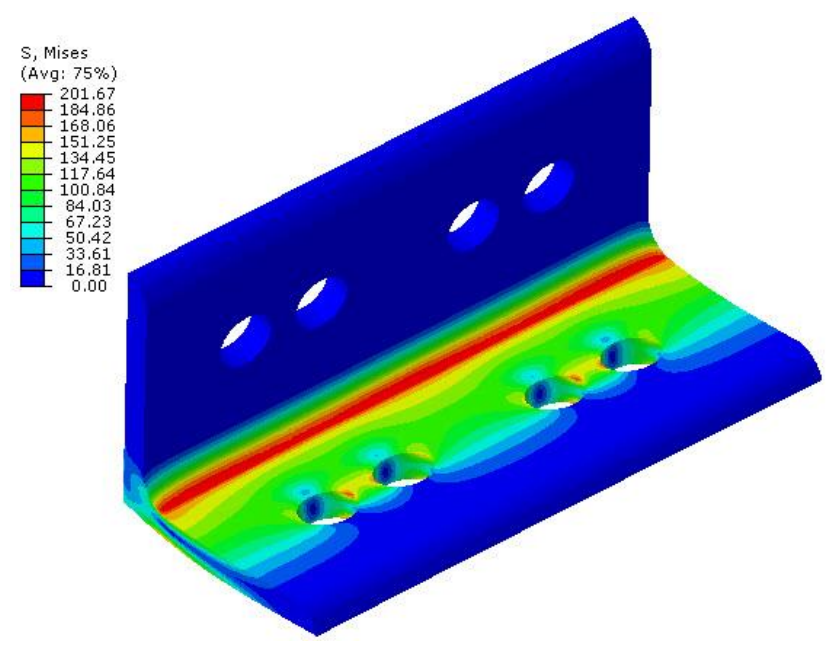

(a) FEM

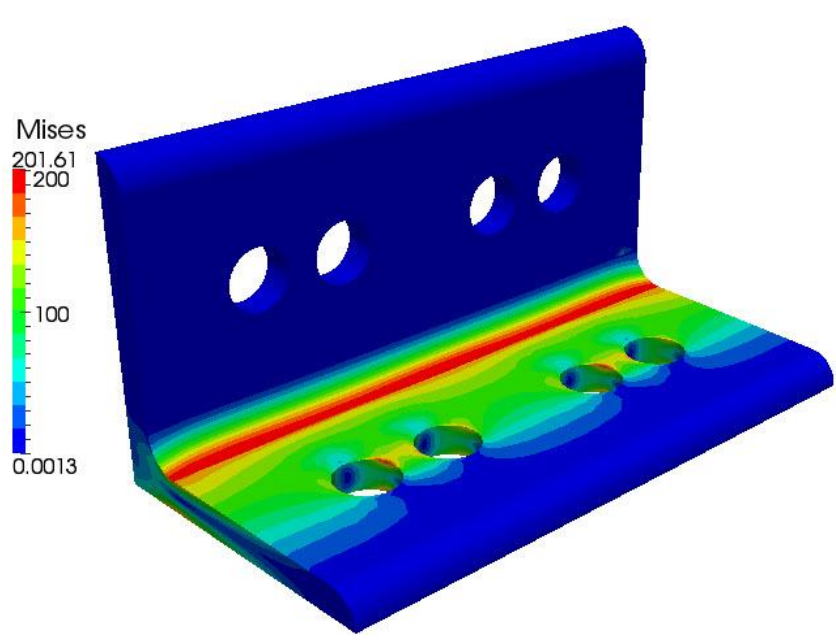

(b) DiBFM

Fig. 14. The Von Mises stress results by the two methods

Table 2. Comparison of the maximum displacement and the maximum Von Mises stress by DiBFM and FEM.

\begin{tabular}{c|ccc}
\hline Result & FEM & DiBFM & err $=\left|\frac{x_{\text {DiBFM }}-x_{\text {FEM }}}{x_{\text {FEM }}}\right| \times 100 \%$ \\
\hline U_max & 2.365 & 2.362 & $0.127 \%$ \\
\hline Von Mises_max & 201.67 & 201.61 & $0.030 \%$ \\
\hline
\end{tabular}

From Fig.13 and Fig.14, it can be clearly seen that the displacement and Von Mises stress by DiBFM are almost coincident with those by FEM. In addition, from Table 2 we can see that the relative errors of the maximum displacement and the maximum Von Mises stress are $0.127 \%$ and $0.030 \%$, respectively. These results demonstrate that the DiBFM can solve the practical problems in engineering with high accuracy.

\section{Conclusions}

In this paper, a dual interpolation boundary face method has been extended to 3D elasticity problems successfully. DiBFM is combined with the dual interpolation method and the boundary face method. The numerical results has demonstrated the high accuracy and convergence of the DiBFM in 3D elasticity problems. In addition, the numerical results show that the DiBFM is suitable for solving the problem of structures with small features, since the traction in DiBFM can be obtained directly without geometric errors. This is a very significant behavior since the maximum 
stress usually occurs at the location of the small features. The behavior will provide more reasonable stress results in numerical analysis. In future work, the DiBFM will be extended to analyze the large-scale complex structures via combining with the fast multipole method or adaptive cross method,.

\section{Acknowledgements}

This work was supported by National Natural Science Foundation of China under grant number 11772125 .

\section{References}

[1] J.M. Zhang, W.C. Lin, Y.Q. Dong, A double-layer interpolation method for implementation of BEM analysis of problems in potential theory, Applied Mathematical Modelling, 51 (2017) 250-269.

[2] J.M. Zhang, W. Lin, Y. Dong, A dual interpolation boundary face method for elasticity problems, Eur. J. Mech. - A/Solids 73 (2019) 500-511.

[3] J.M. Zhang, Y.D. Zhong, Y.Q. Dong, et al. Expanding element interpolation method for analysis of thin-walled structures. Engineering Analysis with Boundary Elements, 2018, 86: 82-88.

[4] J.M. Zhang, X.M. Shu, J. Trevelyan, W.C. Lin, P.F. Chai. A solution approach for contact problems based on the dual interpolation boundary face method. Applied Mathematical Modelling, 70 (2019) 643-658.

[5] J.M. Zhang, Y.Q. Dong, W.C. Lin, et al. A singular element based on dual interpolation BFM for V-shaped notches. Applied Mathematical Modelling, 2019.

[6] G.D Manolis, P.K Banerjee. Conforming versus non-conforming boundary elements in three-dimensional elastostatics. International Journal for Numerical Methods in Engineering, 1986, 23(10): 1885-1904.

[7] Grilli S T, Svendsen I A. Corner problems and global accuracy in the boundary element solution of nonlinear wave flows. Engineering Analysis with Boundary Elements, 1990, 7(4): 178-195.

[8] Rosen D, Cormack DE. On corner analysis in the BEM by the continuation approach. Engineering analysis with boundary elements, 1995;16:53-63.

[9] Thompson J F, Soni B K, Weatherill N P. Handbook of grid generation. CRC press, 1998.

[10] Bern M, Eppstein D, Teng S H. Parallel construction of quadtrees and quality triangulations. International Journal of Computational Geometry \& Applications, 1999, 9(06): 517-532.

[11] J.M. Zhang, X.Y. Qin, X. Han, A boundary face method for potential problems in three dimensions, International Journal for Numerical Methods in Engineering, 80 (2009) 320-337.

[12] M.J Peake, J Trevelyan, Coates G. Extended isogeometric boundary element method (XIBEM) for two-dimensional Helmholtz problems. Computer Methods in Applied Mechanics and Engineering, 2013, 259: 93-102. 
[13] X.Y. Qin, J.M. Zhang, G.Y. Li, et al. An element implementation of the boundary face method for 3D potential problems. Engineering analysis with boundary elements, 2010, 34(11): 934-943.

[14] Gong Y P, Dong C Y. An isogeometric boundary element method using adaptive integral method for 3D potential problems. Journal of Computational and Applied Mathematics, 2017, 319: 141-158.

[15] O.C. Zienkiewicz, R.L. Taylor, J.Z. Zhu, The Finite Element Method: Its Basis and Fundamentals. Elsevier, Amsterdam (2013)

[16] P. Lancaster, K. Salkauskas, Surface generated by moving least squares methods, Mathematics of computation, 37 (1981) 141-158.

[17] C.A Brebbia, J.C.F Telles, L.C Wrobel. Boundary element techniques: theory and applications in engineering. Springer Science \& Business Media, 2012.

[18] J.H. Kane, Boundary Element Analysis in Engineering Continuum Mechanics, Prentice-Hall, Engelwood Cliffs, NJ, 1994.

[19] Zhang Y, Li X, Sladek V, et al. A new method for numerical evaluation of nearly singular integrals over high-order geometry elements in 3D BEM. Journal of Computational and Applied Mathematics, 2015, 277: $57-72$.

[20] Zheng B, Gao X, Zhang C. Radial integration BEM for vibration analysis of two-and three-dimensional elasticity structures. Applied Mathematics and Computation, 2016, 277: 111-126.

[21] Liu Y J, Ye W, Deng Y. On the Identities for Elastostatic Fundamental Solution and Nonuniqueness of the Traction BIE Solution for Multiconnected Domains. Journal of Applied Mechanics, 2013, 80(5): 051012.

[22] Honnor M E, Trevelyan J, Huybrechs D. Numerical evaluation of the two-dimensional partition of unity boundary integrals for Helmholtz problems. Journal of Computational and Applied Mathematics, 2010, 234(6): 1656-1662.

[23] Niu Z, Wendland W L, Wang X, et al. A semi-analytical algorithm for the evaluation of the nearly singular integrals in three-dimensional boundary element methods. Computer methods in applied mechanics and engineering, 2005, 194(9-11): 1057-1074.

[24] Y. Li, J.M. Zhang, G.Z. Xie, Time-domain BEM analysis for three-dimensional elastodynamic problems with initial conditions, Computer Modeling in Engineering and Sciences, 101(3) (2014) 187-206.

[25] W.H. Press, S.A. Teukolsky, W.T. Vetterling, et al. Numerical recipes 3rd edition: The art of scientific computing. Cambridge university press, 2007. 